\title{
Outcomes of extracorporeal cardiopulmonary resuscitation for refractory cardiac arrest in adult cardiac surgery patients
}

\author{
Michael A. Mazzeffi, MD, MPH, ${ }^{\mathrm{a}, \mathrm{b}}$ Pablo G. Sanchez, MD, ${ }^{\mathrm{c}}$ Daniel Herr, MD, ${ }^{\mathrm{b}}$ Eric Krause, MD, JD, \\ Charles F. Evans, MD, ${ }^{c}$ Raymond Rector, CCP, ${ }^{c}$ Brian McCormick, CCP, ${ }^{c}$ Si Pham, MD, \\ Bradley Taylor, MD, MPH, ${ }^{\mathrm{c}}$ Bartley Griffith, $\mathrm{MD},{ }^{\mathrm{c}}$ and Zachary N. Kon, $\mathrm{MD}^{\mathrm{c}}$
}

\begin{abstract}
Background: The role of extracorporeal cardiopulmonary resuscitation (ECPR) in adult cardiac surgery patients with refractory cardiac arrest is uncertain. We hypothesized that ECPR would be associated with better than expected outcomes in this group of patients.

Methods: We conducted a single-center retrospective cohort study of adult cardiac surgery patients who underwent ECPR for refractory cardiac arrest during a 6-year period (2010 to 2015). In-hospital mortality, survival at last follow-up, and cerebral performance category (CPC) were examined as outcomes, and potential risk factors for mortality were explored.
\end{abstract}

Results: Twenty-three patients underwent ECPR when spontaneous circulation did not return with conventional resuscitation. Thirty-day mortality was $65.2 \%$, and in-hospital mortality was $69.6 \%$. Six of the 23 patients $(26.1 \%)$ were discharged with a favorable neurologic outcome, defined as CPC 1 or 2. Most patients who died had multiple organ dysfunction syndrome $(43.8 \%)$, and a smaller number had severe brain injury $(25.0 \%)$. Kaplan-Meier survival analysis suggested age as a critical factor affecting survival $(P=.04$, log-rank test).

Conclusions: ECPR may have a role in younger adult cardiac surgery patients who experience refractory cardiac arrest. Future studies are needed to identify patients who will benefit most from ECPR. (J Thorac Cardiovasc Surg 2016;152:1133-9)

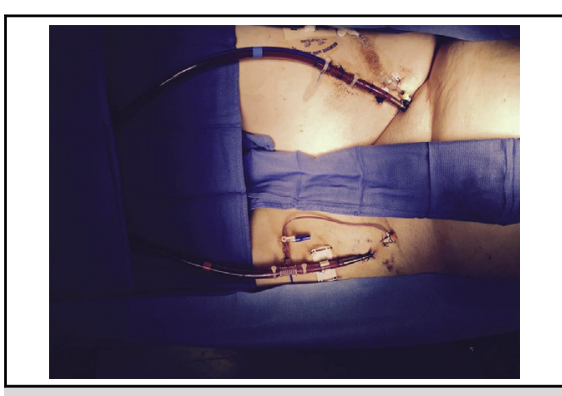

Peripheral venoarterial extracorporeal life support cannulation with a venous cannula in the right femoral vein and an arterial cannula in the left common femoral artery. The distal perfusion cannula, a 6 F sheath, is in the left superficial femoral artery.

\section{Central Message}

ECPR may have a role in younger adult cardiac surgery patients experiencing refractory cardiac arrest.

\section{Perspective}

The role of ECPR in refractory cardiac arrest after adult cardiac surgery is uncertain. Our study results suggest that ECPR may provide better than expected outcomes, particularly in young patients.

See Editorial Commentary page 1140.
Adult in-hospital cardiac arrest remains a significant problem, with more than 200,000 arrests per year. ${ }^{1}$ The majority of these arrests $(80 \%)$ begin as pulseless electrical activity (PEA) or asystole; the remainder, as ventricular arrhythmias. Historically, the mortality rate for in-hospital cardiac arrests has been between $80 \%$ and $90 \%{ }^{2}$

\footnotetext{
From the Departments of ${ }^{\mathrm{a}}$ Anesthesiology and ${ }^{\mathrm{c}}$ Cardiac Surgery, and ${ }^{\mathrm{b}}$ Shock Trauma Critical Care, University of Maryland School of Medicine, Baltimore, Md.

Received for publication Jan 15, 2016; revisions received May 26, 2016; accepted for publication June 8, 2016; available ahead of print July 12, 2016.

Address for reprints: Michael A. Mazzeffi, MD, MPH, Department of Anesthesiology, University of Maryland School of Medicine, 22 South Greene St S11C00, Baltimore, MD 21201 (E-mail: mmazzeffi@anes.umm.edu). $0022-5223 / \$ 36.00$

Copyright (C) 2016 by The American Association for Thoracic Surgery http://dx.doi.org/10.1016/j.jtcvs.2016.06.014
}

Approximately one-third of survivors have a clinically significant neurologic disability at discharge. Fortunately, the last decade has seen modest improvements in mortality rates and neurologic outcomes, ${ }^{2}$ likely due to advances in both the resuscitation process itself and postarrest management.

After cardiac surgery, patients are uniquely susceptible to cardiac arrest, which affects up to $5 \%$ of patients. ${ }^{3}$ This increased vulnerability arises from the nature of

Scanning this QR code will take you to a video for the article.

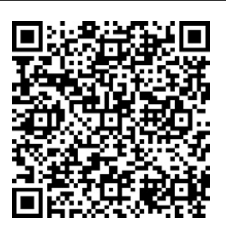




$$
\begin{aligned}
\text { Abbreviations and Acronyms } \\
\text { ACLS }=\text { advanced cardiac life support } \\
\text { AHA }=\text { American Heart Association } \\
\text { CCPR }=\text { conventional cardiopulmonary } \\
\text { resuscitation } \\
\text { CPC }=\text { cerebral performance category } \\
\text { ECLS }=\text { extracorporeal life support } \\
\text { ECPR }=\text { extracorporeal cardiopulmonary } \\
\text { resuscitation } \\
\text { ICU }=\text { intensive care unit } \\
\text { OR }=\text { operating room } \\
\text { PEA }=\text { pulseless electrical activity } \\
\text { ROSC }=\text { return of spontaneous circulation }
\end{aligned}
$$

the procedures performed. After cardiac surgery, the myocardium is more irritable and likely to suffer a lethal arrhythmia related to ischemia during the procedure, reperfusion injury, air embolism, or cardiac tamponade. How centers respond to cardiac arrest after surgery is as variable as the success rate of resuscitation. ${ }^{3}$ The European Association of Cardiothoracic Surgery has adopted a standardized resuscitation algorithm for cardiac surgery patients. This algorithm is similar to the American Heart Association (AHA) advanced cardiac life support (ACLS) algorithms, but includes early sternal reentry and lower bolus doses of vasoactive medications. ${ }^{4,5}$

The use of extracorporeal life support (ECLS) has increased dramatically in recent years, and recent case series and cohort studies suggest that pediatric patients with refractory cardiac arrest may benefit from extracorporeal cardiopulmonary resuscitation (ECPR). ${ }^{6,7}$ In adults, the role for ECPR is less clear and neither European guidelines or AHA guidelines recommend ECPR as part of standard resuscitation. The 2015 update to the AHA's ACLS guidelines notes that there is insufficient evidence to recommend the routine use of ECPR for patients with cardiac arrest, but that ECPR may be considered in selected patients for whom the cause is potentially reversible. $^{8}$

The purpose of the present study was to review our institution's experience with ECPR in cardiac surgery patients who experience refractory cardiac arrest after surgery, a patient population with particularly poor survival. We hypothesized that ECPR would be associated with better than expected outcomes in patients with refractory cardiac arrest.

\section{METHODS \\ Subjects}

The University of Maryland's Institutional Review Board approved this study. We performed a retrospective cohort study of all adult patients who received ECPR for refractory cardiac arrest after cardiac surgery during a 6-year period (2010 to 2015). Patients who had ECPR were identified using our institution's ECLS database. Only patients who had cardiac surgery with cardiopulmonary bypass or transcatheter aortic valve replacement were included. We confirmed that we had captured all cases by reviewing all postsurgical arrests that occurred during the study period in our institutional Society of Thoracic Surgeons database. Refractory arrest was defined as a cardiac arrest in which standard resuscitation did not achieve a return of spontaneous circulation (ROSC) and it was determined that the patient would not survive without ECPR. Our general practice is to initiate the ECPR process if ROSC is not achieved within 10 minutes. For all patients, we recorded the following variables: age, sex, history of diabetes mellitus, history of hypertension, baseline creatinine, preoperative left ventricular ejection fraction, preoperative right ventricular function, type of surgery, cardiopulmonary bypass time during the index operation, postoperative day of the arrest, putative cause of arrest, initial heart rhythm during the arrest, minutes of conventional cardiopulmonary resuscitation (CCPR) before institution of ECPR, and cannulation scheme.

\section{ECLS and Resuscitation Details}

Our center has a well-established ECLS program, and more than 300 patients received ECLS for various indications during the study period. The typical ECLS circuit at our center includes a Rotaflow centrifugal pump (Maquet, Fairfield, NJ) and Quadrox oxygenator (Maquet). Heparin is used for anticoagulation, with a target activated partial thromboplastin time of 60 to 80 seconds or an activated clotting time of 180 to 200 seconds in patients receiving venoarterial ECLS.

Cardiac surgeons performed all cannulations during the study period. The decision of central or peripheral cannulation was made by the attending surgeon depending on which procedure was deemed the most expedient. Before initiation of ECLS, resuscitation was performed according to modified ACLS guidelines that included the European Association of Cardiothoracic Surgery's recommendation for early sternal reentry in patients at $<10$ days postsurgery. Post-ECPR care was provided by a multidisciplinary team that included intensivists, cardiac surgeons, nurses, perfusionists, and respiratory therapists. ECLS flows were targeted to achieve a cardiac index of $\geq 2.2 \mathrm{~L} / \mathrm{min} / \mathrm{m}^{2}$. All patients were supported with a low- to moderate-dose inotropic agent (typically epinephrine) to promote left ventricular ejection and prevent cardiac thrombus formation. Mean arterial pressure was targeted at 65 to $80 \mathrm{~mm} \mathrm{Hg}$.

Transthoracic echocardiography was performed approximately every 2 to 3 days during ECLS to confirm left ventricular decompression and evaluate cardiac recovery. Three patients had an intra-aortic balloon placed for augmented left ventricular unloading. No patient underwent mechanical left ventricular venting, atrial septostomy, or placement of an Impella circulatory assist device (Abiomed, Danvers, Mass), because these are not standard practices in patients receiving ECPR at our center. ECLS weaning trials were attempted every 2 to 3 days. Ventricular function was assessed by echocardiography, and cardiac output was measured by thermodilution with a pulmonary artery catheter. Patients who maintained a cardiac index of $>2.0 \mathrm{~L} / \mathrm{min} / \mathrm{m}^{2}$ and a mean arterial pressure of $\geq 65 \mathrm{~mm} \mathrm{Hg}$ on a single inotropic agent with minimal ECLS support $(\leq 2 \mathrm{~L} / \mathrm{min}$ ) were considered ready for a formal weaning trial in the operating room (OR).

\section{Temperature Management}

There was no formal protocol for temperature management in ECPR recipients during the study period. In 17 patients, temperature was maintained at $36^{\circ} \mathrm{C}$ for the first 24 hours after arrest and then kept below $38^{\circ} \mathrm{C}$ for the remainder of ECLS. Six patients were cooled to $32^{\circ} \mathrm{C}$ to $34^{\circ} \mathrm{C}$ during the first 24 hours after arrest and then maintained below $38^{\circ} \mathrm{C}$ for the remainder of ECLS. 


\section{Outcome Variables}

The following outcome variables were recorded: 30-day mortality, in-hospital mortality, survival at last follow-up, acute renal failure requiring renal replacement therapy, total ECLS days, successful decannulation (defined as off ECLS for $>48$ hours), postdecannulation cardiac support, cause of death, and neurologic outcome. Specifically, for neurologic outcome, we recorded whether brain imaging was performed, whether anoxic brain injury or brain death occurred, and the cerebral performance category (CPC) at the time of death or hospital discharge. Brain death was defined as irreversible loss of all cortical and brainstem function.

\section{Statistical Analysis}

Statistical analyses were performed with SAS 9.3 (SAS Institute, Cary, NC). Continuous variables that were normally distributed are reported as mean \pm standard deviation, and skewed variables are reported as median and interquartile range. Categorical variables are reported as the number and percentage of patients.

Survival analyses were performed using Kaplan-Meier analysis. Survival at last follow-up was modeled for all patients in the cohort. In addition, survival was also modeled after stratification into 3 age groups ( $<50$ years, 50 to 70 years, and $>70$ years) and after stratification by CCPR time before initiation of ECPR ( $<30$ minutes or $\geq 30$ minutes). The log-rank test was used to compare survival between groups. A $P$ value $<.05$ was considered to indicate statistical significance.

Finally, patients were stratified based on potential risk factors for mortality, and a 2-tailed $\chi^{2}$ test was applied to test for significant differences between groups. The relationships between CCPR time and in-hospital mortality and between age and in-hospital mortality, considered of particular interest, were also modeled using bivariate logistic regression.

\section{RESULTS}

A total of 5444 patients underwent cardiac surgery during the study period and were screened for inclusion in the present study. Of these, 191 experienced postoperative cardiac arrest. Twenty-three patients received ECPR for refractory arrest; characteristics of these patients are summarized in Table 1 . The mean patient age was 57 years (range, 34 to 86 years). The most frequent cause of arrest was cardiac tamponade, and the majority of patients presented with PEA. All of the patients with tamponade underwent a repeat sternotomy. The 3 patients with coronary artery obstruction due to a surgical complication returned to the OR for correction. Most of the patients who received ECPR were in the intensive care unit (ICU), with a small number in the OR or on the telemetry floor. The 5 ECPR cannulations done on the telemetry floor were central. One peripheral and 2 central cannulations were performed in the OR, and 8 peripheral and 7 central cannulations were done in the ICU.

Table 2 presents the outcome variables for patients in the cohort. Thirty-day mortality was $65.2 \%$, and inhospital mortality was $69.6 \%$. Three patients suffered early death due to either hemorrhage or the inability to regain a perfusing cardiac rhythm. The median number of ECLS days was 3 (range, 0 to 14 days). Figure 1
TABLE 1. Patient characteristics $(\mathbf{n}=\mathbf{2 3})$

\begin{tabular}{|c|c|}
\hline Variable & Value \\
\hline Age, $y$, mean $\pm S D$ & $57 \pm 15$ \\
\hline Male sex, n (\%) & $14(60.9)$ \\
\hline Diabetes mellitus, n (\%) & $5(21.7)$ \\
\hline Hypertension, n (\%) & $18(78.3)$ \\
\hline Baseline creatinine, $\mathrm{mg} / \mathrm{dL}$, median (IQR) & $1.2(0.9-2.0)$ \\
\hline $\begin{array}{l}\text { Preoperative left ventricular ejection fraction, } \% \text {, } \\
\text { median (IQR) }\end{array}$ & $50(40-55)$ \\
\hline \multicolumn{2}{|l|}{ Preoperative right ventricular dysfunction, n (\%) } \\
\hline None & $14(60.9)$ \\
\hline Mild & $2(8.7)$ \\
\hline Moderate & $3(13.0)$ \\
\hline Severe & $4(17.4)$ \\
\hline \multicolumn{2}{|l|}{ Type of surgery, n (\%) } \\
\hline Isolated CABG & $7(30.4)$ \\
\hline Single valve & $5(21.8)$ \\
\hline Aortic replacement & $7(30.4)$ \\
\hline $\begin{array}{l}\text { Other (heart transplant, TAVR, VSD closure, } \\
\text { multivalve) }\end{array}$ & $4(17.4)$ \\
\hline Cardiopulmonary bypass time, min, median (IQR) & $160(103-254)$ \\
\hline Postoperative day of arrest, median (IQR) & $2(0-5)$ \\
\hline \multicolumn{2}{|l|}{ Putative cause of arrest, $\mathrm{n}(\%)$} \\
\hline Tamponade & $4(17.4)$ \\
\hline Right ventricular failure & $3(13.0)$ \\
\hline Coronary artery obstruction and ischemia & $3(13.0)$ \\
\hline Respiratory arrest & $2(8.7)$ \\
\hline Biventricular failure & $1(4.4)$ \\
\hline Severe acidosis & $1(4.4)$ \\
\hline Unclear etiology & $9(39.1)$ \\
\hline \multicolumn{2}{|l|}{ Presenting rhythm, n (\%) } \\
\hline PEA & $15(65.2)$ \\
\hline Asystole & $2(8.7)$ \\
\hline Ventricular arrhythmia & $6(26.1)$ \\
\hline $\begin{array}{l}\text { Duration of CCPR before initiation of ECPR, min, } \\
\text { median (IQR) }\end{array}$ & $31(15-52)$ \\
\hline \multicolumn{2}{|l|}{ Location of cardiac arrest, $\mathrm{n}(\%)$} \\
\hline Operating room & $3(13.0)$ \\
\hline Intensive care unit & $15(65.2)$ \\
\hline Telemetry floor & $5(21.8)$ \\
\hline \multicolumn{2}{|l|}{ Type of cannulation, $\mathrm{n}(\%)$} \\
\hline Central & $14(60.9)$ \\
\hline Peripheral & $9(39.1)$ \\
\hline
\end{tabular}

$S D$, Standard deviation; $I Q R$, interquartile range; $C A B G$, coronary artery bypass grafting; TAVR, transcatheter aortic valve replacement; $V S D$, ventricular septal defect; $P E A$, pulseless electrical activity; $C C P R$, conventional cardiopulmonary resuscitation; $E C P R$, extracorporeal cardiopulmonary resuscitation.

shows the survival probability over time for all ECPR recipients. Figure 2 shows the survival probability after stratification by age, and Figure 3 shows the survival probability after stratification by duration of CCPR before initiation of ECPR. Younger age was associated with improved survival $(P=.04)$, whereas no association was found between CCPR time $<30$ minutes and survival $(P=.29)$.

Four of the 20 patients who survived the initial resuscitation had an adverse neurologic outcome (3 with 
TABLE 2. Patient outcomes $(n=23)$

\begin{tabular}{|c|c|}
\hline Variable & Value \\
\hline 30-d mortality, n (\%) & $15(65.2)$ \\
\hline In-hospital mortality, n (\%) & $16(69.6)$ \\
\hline \multicolumn{2}{|l|}{ Early death $(<3 \mathrm{~h}), \mathrm{n}(\%)$} \\
\hline Hemorrhage & $2(8.7)$ \\
\hline Unable to regain perfusing rhythm & $1(4.2)$ \\
\hline \multicolumn{2}{|l|}{ Neurologic outcomes, n (\%) } \\
\hline \multicolumn{2}{|l|}{ Temperature management } \\
\hline $32^{\circ} \mathrm{C}-34^{\circ} \mathrm{C}$ for the first $24 \mathrm{~h}$ & $6(26.1)$ \\
\hline $36^{\circ} \mathrm{C}$ for the first $24 \mathrm{~h}$ & $17(73.9)$ \\
\hline Underwent neurologic imaging & $8(34.8)$ \\
\hline Survival with $\mathrm{CPC} \leq 2$ & $6(26.1)$ \\
\hline \multicolumn{2}{|l|}{$\mathrm{CPC}$ in all patients } \\
\hline 1 & $1(4.3)$ \\
\hline 2 & $5(21.7)$ \\
\hline 3 & $10(43.5)$ \\
\hline 4 & $1(4.3)$ \\
\hline 5 & $3(13.1)$ \\
\hline Unable to assess due to early death & $3(13.1)$ \\
\hline \multicolumn{2}{|l|}{ Severe brain injury } \\
\hline Diffuse anoxic brain injury & $1(4.2)$ \\
\hline Brain death & $3(12.5)$ \\
\hline $\begin{array}{l}\text { New renal failure requiring continuous renal } \\
\text { replacement therapy, } \mathrm{n}(\%)\end{array}$ & $11(47.8)$ \\
\hline Total ECLS days, median (IQR) & $3(1-8)$ \\
\hline Decannulation for $\geq 48 \mathrm{~h}, \mathrm{n}(\%)$ & $14(60.8)$ \\
\hline \multicolumn{2}{|l|}{ Postdecannulation support, $\mathrm{n}(\%)$} \\
\hline Right ventricular assist device & $1(4.3)$ \\
\hline Biventricular assist device & $1(4.3)$ \\
\hline Inotropes until death, without mechanical support & $14(60.9)$ \\
\hline Weaning from inotropic therapy & $7(30.5)$ \\
\hline \multicolumn{2}{|l|}{ Cause of death, $\mathrm{n}(\%)$} \\
\hline Multiple organ dysfunction syndrome & $7(43.8)$ \\
\hline Brain injury & $4(25.0)$ \\
\hline Hemorrhage & $1(6.2)$ \\
\hline Sepsis & $3(18.8)$ \\
\hline Repeat arrest & $1(6.2)$ \\
\hline
\end{tabular}

$C P C$, Cerebral performance category, $E C L S$, extracorporeal life support; $I Q R$, interquartile range.

brain death and 1 with diffuse anoxic brain injury). All but 1 survivor had a favorable neurologic outcome, defined as a CPC of 1 or 2; that survivor, who had a CPC of 3 , required persistent rehabilitation care after hospital discharge and died approximately 8 months after receiving ECPR.

Most of the patients who were decannulated were supported only with inotropic agents after completion of ECLS; however, 2 patients were supported with a temporary extracorporeal ventricular assist device. Both of these patients subsequently died. The most frequent cause of death was multiple organ dysfunction syndrome, followed by brain injury and sepsis.

Table 3 presents the frequencies of in-hospital death after stratification by potential risk factors. The only potential risk factors with a significant association with mortality

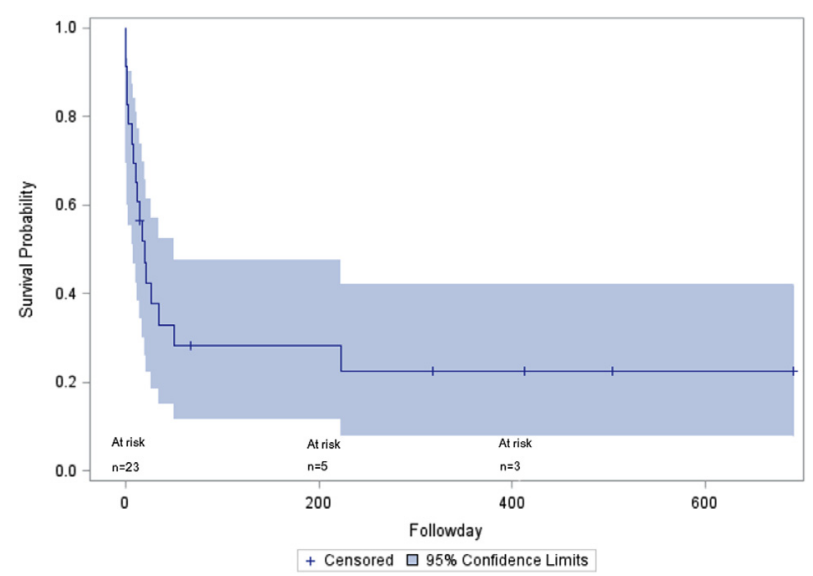

FIGURE 1. Kaplan-Meier analysis showing survival at last follow-up. The shaded area represents the $95 \%$ confidence interval.

were age $\geq 50$ years $(P=.005)$ and cause of cardiac arrest other than tamponade $(P=.03)$. The odds ratio for in-hospital death with each 1-year increase in age was 1.12 (95\% confidence interval, 1.01 to $1.24 ; P=.03$ ), and the odds ratio for in-hospital death with each CCPR minute before ECPR was 1.00 (95\% confidence interval, 0.97 to $1.05 ; P=.77)$.

\section{DISCUSSION}

In-hospital cardiac arrest carries a grim prognosis, with only $10 \%$ to $20 \%$ of patients surviving hospitalization. ${ }^{2,9}$ Factors previously associated with survival include younger age, arrest occurring somewhere other than the general hospital floor or the emergency room, short duration of CCPR, no intubation during arrest, surgical patient versus medical patient, lack of existing malignancy, and no prearrest vasopressor therapy. ${ }^{10,11}$

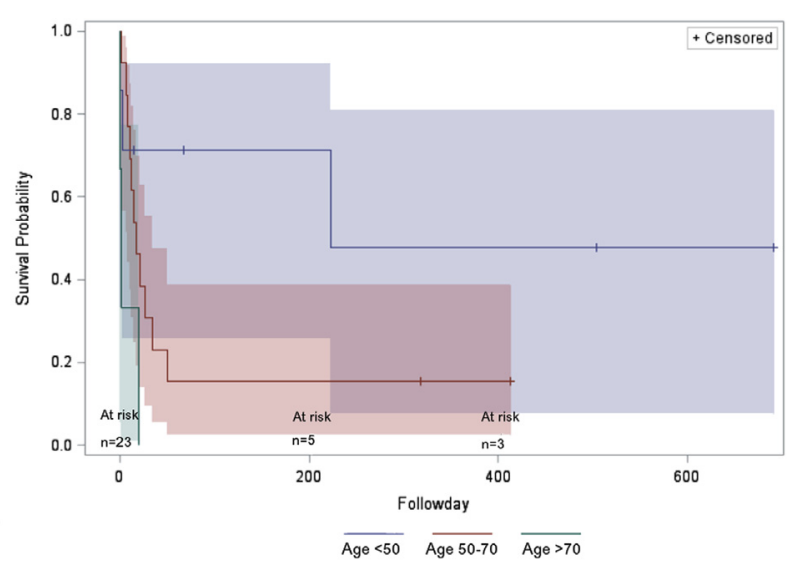

FIGURE 2. Kaplan-Meier analysis showing survival at last follow-up after stratification by age group. The shaded area represents the $95 \%$ confidence interval. 


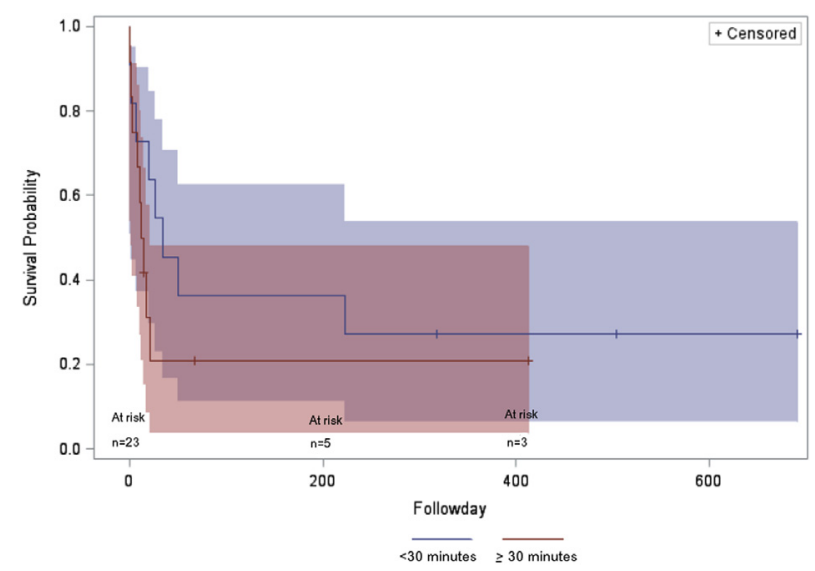

FIGURE 3. Kaplan-Meier analysis showing survival at last follow-up after stratification by duration of CCPR before initiation of ECPR. The shaded area represents the $95 \%$ confidence interval.

For patients who experience a cardiac arrest, there appears to be a decrement in functional survival for each minute of CCPR, and most patients who experience functional recovery exhibit ROSC within 16 minutes of initiation of CCPR. ${ }^{12}$ Despite these data, there remains a high variability in the duration of CCPR between centers, and centers that perform longer CCPR appear to have better rates of overall survival to discharge. ${ }^{13}$ ECLS has been used increasingly in recent years for patients with refractory cardiopulmonary failure, and recent studies suggest that ECPR may improve survival and neurologic outcomes in pediatric patients who experience cardiac arrest. ${ }^{6,7,14}$ Data supporting the use of ECPR in adults is less robust, however, particularly in patients who experience arrest after cardiac surgery.

ECPR may improve survival in patients with refractory cardiac arrest for several reasons. First, it allows for more rapid and stable restoration of mean arterial blood pressure and cerebral blood flow in patients who experience refractory arrest. We believe that this is particularly true at an experienced center in which cannulation can be performed rapidly and there is minimal interruption in chest compressions. In the present study, the median time from the start of arrest to full-flow ECLS was $31 \mathrm{mi}-$ nutes at our center. Unfortunately, our retrospective review is unable to identify the exact time point when the decision was made to initiate ECLS, and thus we cannot quantify how many minutes were required for cannulation.

Another potential benefit of ECPR in refractory cardiac arrest is that it allows for more predictable postarrest temperature management. Because ECLS circuits contain a heat exchanger, sustained fever after an arrest is very uncommon in ECPR recipients. This may decrease brain injury and improve overall outcome. Finally, patients started on ECLS exhibit an immediate reduction in
TABLE 3. In-hospital mortality stratified by potential risk factors $(\mathbf{n}=\mathbf{2 3})$

\begin{tabular}{|c|c|c|}
\hline Variable & $\begin{array}{c}n / N \\
(\% \text { mortality })\end{array}$ & $\begin{array}{c}P \\
\text { value }\end{array}$ \\
\hline Diabetes mellitus & $5 / 5(100)$ & .09 \\
\hline No diabetes mellitus & $11 / 18(61.1)$ & \\
\hline Hypertension & $13 / 18(72.2)$ & .60 \\
\hline No hypertension & $3 / 5(60.0)$ & \\
\hline Baseline plasma creatinine $\geq 2.0 \mathrm{mg} / \mathrm{dL}$ & $5 / 7(71.4)$ & .90 \\
\hline Baseline plasma creatinine $<2.0 \mathrm{mg} / \mathrm{dL}$ & $11 / 16(68.8)$ & \\
\hline $\begin{array}{l}\text { Baseline left ventricular ejection } \\
\text { fraction } \leq 30 \%\end{array}$ & $4 / 5(80.0)$ & .57 \\
\hline $\begin{array}{l}\text { Baseline left ventricular ejection } \\
\text { fraction }>30 \%\end{array}$ & $12 / 18(66.7)$ & \\
\hline PEA or asystole & $12 / 17(70.6)$ & .86 \\
\hline Ventricular arrhythmia & $4 / 6(66.7)$ & \\
\hline Arrest not in first $48 \mathrm{~h}$ & $8 / 9(88.9)$ & .11 \\
\hline Arrest in first $48 \mathrm{~h}$ & $8 / 14(57.1)$ & \\
\hline Arrest on telemetry & $5 / 5(100.0)$ & .09 \\
\hline $\begin{array}{l}\text { Arrest in operating room or } \\
\text { intensive care unit }\end{array}$ & $11 / 18(61.1)$ & \\
\hline Central cannulation & $11 / 14(78.6)$ & .24 \\
\hline Peripheral cannulation & $5 / 9(55.6)$ & \\
\hline $\mathrm{CCPR} \geq 30 \mathrm{~min}$ before ECPR & $9 / 12(75.0)$ & .55 \\
\hline CCPR $<30 \mathrm{~min}$ before ECPR & $7 / 11(63.6)$ & \\
\hline Age $\geq 50 y$ & $14 / 16(87.5)$ & .005 \\
\hline Age $<50$ y & $2 / 7(28.6)$ & \\
\hline Isolated coronary artery bypass grafting & $4 / 7(57.1)$ & .27 \\
\hline Isolated single valve & $5 / 5(100)$ & \\
\hline Aortic replacement & $4 / 7(57.1)$ & \\
\hline $\begin{array}{l}\text { Other (heart transplant, TAVR, } \\
\text { VSD closure, multivalve) }\end{array}$ & $3 / 4(75.0)$ & \\
\hline Tamponade causing arrest & $1 / 4(25.0)$ & .03 \\
\hline Other cause & $15 / 19(78.9)$ & \\
\hline
\end{tabular}

$P E A$, Pulseless electrical activity; $C C P R$, conventional cardiopulmonary resuscitation; $E C P R$, extracorporeal cardiopulmonary resuscitation; TAVR, transcatheter aortic valve replacement; $V S D$, ventricular septal defect.

preload, as venous blood is diverted away from the heart. This in turn decreases myocardial oxygen consumption and may allow injured myocardium time to rest and recover.

A recent observational study from Japan that included data from 46 hospitals and 234 ECPR recipients reported $68 \%$ survival in patients who experienced out-of-hospital cardiac arrest and received ECPR for resuscitation. However, that study reported 24-hour survival, not survival to hospital discharge. Moreover, there was a high rate of brain injury, with only $12 \%$ of patients having a favorable neurologic outcome at 6 months. ${ }^{15}$ Another recent study compared outcomes in 2 groups of patients who sustained in-hospital cardiac arrest, 1 group who received ECPR and another group who received CCPR, using a propensity score approach to control for differences between the 2 groups. ${ }^{16}$ In that study, which included 59 ECPR recipients (22 surgically treated and 37 medically treated), survival to 


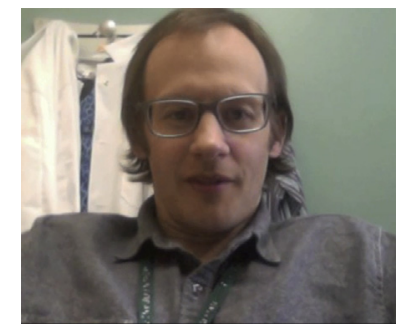

VIDEO 1. The study's lead author speaks about the study's purpose, results, and implications of its findings. Video available at http://www. jtcvsonline.org/article/S0022-5223(16)30644-4/addons.

discharge was $28.8 \%$ in the ECPR group and only $12.3 \%$ in the CCPR group. Two other recent propensity score-matched cohorts found similar benefits in patients receiving ECPR compared with those receiving CCPR. ${ }^{17,18}$

None of the previously mentioned studies evaluated only post-cardiac surgery patients. The sole study, to our knowledge, that has analyzed this population is a study by Zhao et al, who reported the results of ECPR in 24 patients who experienced cardiac arrest after cardiac surgery. ${ }^{19}$ In their study, $33 \%$ of patients survived to hospital discharge with a 4-day average duration of ECLS. The authors did not report variables associated with survival or good neurologic outcome, but $50 \%$ of the patients who died had severe neurologic injury. In a recent case series reported by Anselmi et al, ${ }^{20}$ the rate of brain death was closer to ours at $24.5 \%$, and the mean duration of low flow before the initiation of ECPR was also similar to ours at 47 minutes.

Our data are consistent with the findings of Zhao et al, in that $30 \%$ of our patients survived to discharge. However, our patient mix is quite different than theirs, with only $30 \%$ of our patients having undergone coronary artery bypass graft surgery, compared with $83 \%$ of theirs. We believe that our patient population is more consistent with the current case mix at most major cardiac centers today. Furthermore, our cohort appears to have had a lower rate of major neurologic injury, with only 4 patients experiencing a devastating brain injury. Although our cohort is of modest size, it demonstrates that age has a critical effect on survival, and that patients with tamponade may have better survival compared with patients with other causes of cardiac arrest. This is perhaps the most important aspect of our study, because ECLS is incredibly resource-intensive and costly, ${ }^{21}$ and its effective incorporation into resuscitation algorithms necessitates identification of those patients who are most likely to benefit from it. In our cohort, no patient received ECLS for more than 14 days, suggesting that effective triaging of ECPR recipients can be done without a protracted course, or that patients will declare a path toward recovery or death rather quickly.
Interestingly, unlike in the study by Zhang et al, in our study the duration of CCPR before initiation of ECPR did not have a significant association with survival at last follow-up or in-hospital mortality. We believe that this is because the majority of patients in our study were resuscitated in the ICU or OR with early arrest recognition, minimal interruption of chest compressions, and invasive monitoring (eg, arterial line) allowing for continuous blood pressure measurement during CCPR. There was no statistically significant difference in mortality between patients who had central cannulation and those with peripheral cannulation, but as our program has evolved, peripheral cannulation has become the preferred methodology, and we are currently in the process of devising a more formal protocol for ECPR for patients who experience refractory cardiac arrest. We believe that peripheral cannulation is rapid, is highly reproducible, and allows for less interruption in chest compressions.

This study has several important limitations to consider. First, it is retrospective in nature, and resuscitation data were obtained from records that might have been incomplete or inaccurate in terms of event timing. Second, our cohort is of modest size, and resuscitation care was provided by different physicians and nurses, which might have affected patient outcomes. Third, we acknowledge that there is a high likelihood of selection bias, considering that the patients who received ECPR were likely to have been identified as appropriate candidates by the attending surgeons involved in their care. Fourth, the study had no control group for comparing mortality and neurologic outcomes. We did query our institutional Society of Thoracic Surgeons database, which showed a $58 \%$ rate of in-hospital mortality for all patients experiencing cardiac arrest after cardiac surgery at our center between 2010 and 2015. However, this number represents some patients with easily treated arrest, whereas our cohort included only patients with refractory arrest. Finally, our study results might not be generalizable to other medical centers, given the varying survival by level of experience with ECLS and ECPR, as well as the differences in resuscitation protocols and postresuscitation care.

This study also has some important strengths. Our cohort is one of the few cohorts of adult cardiac surgery patients who received ECPR for refractory cardiac arrest reported in the literature to date. In addition, it includes only patients with long periods of cardiac arrest who likely would have died without ECPR. Finally, this is one of the only studies to evaluate potential variables associated with mortality in cardiac surgery patients receiving ECPR.

In summary, at an experienced ECLS center, ECPR for refractory cardiac arrest in cardiac surgery patients was associated with a better than expected survival rate, with $30 \%$ of patients surviving to discharge and $85.7 \%$ of 
survivors having a favorable neurologic outcome. Age appeared to have a critical impact on survival and is an important variable that should be considered when ECPR is used. Future studies are needed to confirm our findings and better delineate which patients may benefit from ECPR when refractory cardiac arrest occurs after surgery. Once the patients who are most likely to benefit have been identified, a multicenter clinical trial should be strongly considered.

\section{Conflict of Interest Statement}

Authors have nothing to disclose with regard to commercial support.

\section{References}

1. Graham R, McCoy MA, Schultz AM, eds. Strategies to improve cardiac arrest survival: a time to act. Washington (DC): National Academies Press; 2015.

2. Girotra S, Nallamothu BK, Spertus JA, Li Y, Krumholz HM, Chan PS. Trends in survival after in-hospital cardiac arrest. N Engl J Med. 2012; 367:1912-20

3. LaPar DJ, Ghanta RK, Kern JA, Crosby IK, Rich JB, Speir AM, et al. Hospital variation in mortality from cardiac arrest after cardiac surgery: an opportunity for improvement? Ann Thorac Surg. 2014;98:534-40.

4. Field JM, Hazinski MF, Sayre MR, Chameides L, Schexnayder SM, Hemphill R, et al. Part 1: Executive summary: 2010 American Heart Association guidelines for cardiopulmonary resuscitation and emergency cardiovascular care. Circulation. 2010;122(18 Suppl 3):S640-56.

5. Dunning J, Fabbri A, Kolh PH, Levine A, Lockowandt U, Mackay J, et al. Guideline for resuscitation in cardiac arrest after cardiac surgery. Eur $J$ Cardiothorac Surg. 2009;36:3-28.

6. Prodhan P, Fiser RT, Dyamenahalli U, Gossett J, Imamura M, Jaquiss RD, et al. Outcomes after extracorporeal cardiopulmonary resuscitation (ECPR) following refractory pediatric cardiac arrest in the intensive care unit. Resuscitation. 2009; 80:1124-9.

7. Wolf MJ, Kanter KR, Kirshbom PM, Kogon BE, Wagoner SF. Extracorporeal cardiopulmonary resuscitation for pediatric cardiac patients. Ann Thorac Surg. 2012:94:874-9.

8. Neumar RW, Shuster M, Callaway CW, Gent LM, Atkins DL, Bhanji F, et al. Part 1: Executive summary: 2015 American Heart Association guidelines update for cardiopulmonary resuscitation and emergency cardiovascular care. Circulation. 2015;132(18 Suppl 2):S315-67.

9. Johnson NJ, Salhi RA, Abella BS, Neumar RW, Gaieski DF, Carr BG. Emergency department factors associated with survival after sudden cardiac arrest. Resuscitation. 2013;84:292-7.
10. Tortolani AJ, Risucci DA, Rosati RJ, Dixon R. In-hospital cardiopulmonary resuscitation: patient, arrest, and resuscitation factors associated with survival Resuscitation. 1990;20:115-28.

11. Larkin GL, Copes WS, Nathanson BH, Kaye W. Pre-resuscitation factors associated with mortality in 49,130 cases of in-hospital cardiac arrest: a report from the National Registry for Cardiopulmonary Resuscitation. Resuscitation. 2010;81:302-11

12. Reynolds JC, Frisch A, Rittenberger JC, Callaway CW. Duration of resuscitation efforts and functional outcome after out-of-hospital cardiac arrest: when should we change to novel therapies? Circulation. 2013;128:2488-94.

13. Goldberger ZD, Chan PS, Berg RA, Kronick SL, Cooke CR, Lu M, et al Duration of resuscitation efforts and survival after in-hospital cardiac arrest: an observational study. Lancet. 2012;380:1473-81.

14. Maxwell BG, Powers AJ, Sheikh AY, Lee PH, Lobato RL, Wong JK. Resource use trends in extracorporeal membrane oxygenation in adults: an analysis of the Nationwide Inpatient Sample 1998-2009. J Thorac Cardiovasc Surg. 2014 148:416-21.

15. Sakamoto T, Morimura N, Nagao K, Asai Y, Yokota H, Nara S, et al Extracorporeal cardiopulmonary resuscitation versus conventional cardiopulmonary resuscitation in adults with out-of-hospital cardiac arrest: a prospective observational study. Resuscitation. 2014;85:762-8.

16. Chen YS, Lin JW, Yu HY, Ko WJ, Jerng JS, Chang WT, et al. Cardiopulmonary resuscitation with assisted extracorporeal life-support versus conventional cardiopulmonary resuscitation in adults with in-hospital cardiac arrest: an observational study and propensity analysis. Lancet. 2008;372: 554-61.

17. Blumenstein J, Leick J, Liebetrau C, Kempfert J, Gaede L, Groß S, et al Extracorporeal life support in cardiovascular patients with observed refractory in-hospital cardiac arrest is associated with favourable short and long-term outcomes: a propensity-matched analysis. Eur Heart J Acute Cardiovasc Care. October 26, 2015 [Epub ahead of print].

18. Shin TG, Choi JH, Jo IJ, Sim MS, Song HG, Jeong YK, et al. Extracorporeal cardiopulmonary resuscitation in patients with inhospital cardiac arrest: a comparison with conventional cardiopulmonary resuscitation. Crit Care Med. 2011;39:1-7.

19. Zhao Y, Xing J, Du Z, Liu F, Jia M, Hou X. Extracorporeal cardiopulmonary resusciation for adult patients who underwent post-cardiac surgery. Eur J Med Res. 2015;20:83.

20. Anselmi A, Flécher E, Corbineau H, Langanay T, Le Bouquin V, Bedossa M et al. Survival and quality of life after extracorporeal life support for refractory cardiac arrest: a case series. J Thorac Cardiovasc Surg. 2015;150: 947-54.

21. Oude Lansink-Hartgring A, van den Hengel B, van der Bij W, Erasmus ME, Mariani MA, Rienstra M, et al. Hospital costs of extracorporeal life support therapy. Crit Care Med. 2016;44:717-23.

Key Words: resuscitation, ECMO, cardiac arrest, cardiac surgery 